OPEN ACCESS

Edited by: Wen-Lung Ma,

China Medical University, Taiwan

Reviewed by:

Ravindra Deshpande,

Wake Forest School of Medicine,

United States

Aihua Zhang,

Heilongjiang University of Chinese

Medicine, China

*Correspondence:

Yang Zhao

yangzhaotcm@njucm.edu.cn

Desong Kong

kongds@njucm.edu.cn

Zhimin Fan

fanzm@njucm.edu.cn

${ }^{\dagger}$ These authors have contributed equally to this work and share first authorship

Specialty section: This article was submitted to

Gastrointestinal Cancers,

a section of the journal

Frontiers in Oncology

Received: 23 June 2021 Accepted: 18 October 2021 Published: 04 November 2021

Citation:

Zhu G, Wang Y, Wang W, Shang F, Pei B, Zhao $Y$, Kong $D$ and Fan $Z$ (2021) Untargeted GC-MS-Based Metabolomics for Early Detection of Colorectal Cancer.

Front. Oncol. 11:729512. doi: 10.3389/fonc.2021.729512

\section{Untargeted GC-MS-Based Metabolomics for Early Detection of Colorectal Cancer}

\author{
Guoxue Zhu ${ }^{1 \dagger}$, Yi Wang ${ }^{2 \dagger}$, Wang Wang ${ }^{3 \dagger}$, Fang Shang ${ }^{2}$, Bin $\mathrm{Pei}^{2}$, Yang Zhao ${ }^{1 *}$, \\ Desong Kong ${ }^{4 *}$ and Zhimin Fan ${ }^{2 *}$
}

\begin{abstract}
${ }^{1}$ Department of Neurology, Nanjing Hospital of Chinese Medicine Affiliated to Nanjing University of Chinese Medicine, Nanjing University of Chinese Medicine, Nanjing, China, ${ }^{2}$ Department of Anorectal Medicine, Nanjing Hospital of Chinese Medicine Affiliated to Nanjing University of Chinese Medicine, Nanjing University of Chinese Medicine, Nanjing, China, ${ }^{3}$ School of Medicine \& Holistic Integrative Medicine, Nanjing University of Chinese Medicine, Nanjing, China, ${ }^{4}$ Chinese Medicine Modernization and Big Data Research Center, Nanjing Hospital of Chinese Medicine Affiliated to Nanjing University of Chinese Medicine, Nanjing University of Chinese Medicine, Nanjing, China
\end{abstract}

Background: Colorectal cancer $(\mathrm{CRC})$ is one of the most common malignant gastrointestinal cancers in the world with a 5 -year survival rate of approximately $68 \%$. Although researchers accumulated many scientific studies, its pathogenesis remains unclear yet. Detecting and removing these malignant polyps promptly is the most effective method in $\mathrm{CRC}$ prevention. Therefore, the analysis and disposal of malignant polyps is conducive to preventing CRC.

Methods: In the study, metabolic profiling as well as diagnostic biomarkers for CRC was investigated using untargeted GC-MS-based metabolomics methods to explore the intervention approaches. In order to better characterize the variations of tissue and serum metabolic profiles, orthogonal partial least-square discriminant analysis was carried out to further identify significant features. The key differences in $t_{R}-m / z$ pairs were screened by the S-plot and VIP value from OPLS-DA. Identified potential biomarkers were leading in the KEGG in finding interactions, which show the relationships among these signal pathways.

Results: Finally, 17 tissue and 13 serum candidate ions were selected based on their corresponding retention time, $\mathrm{p}$-value, $\mathrm{m} / \mathrm{z}$, and VIP value. Simultaneously, the most influential pathways contributing to $\mathrm{CRC}$ were inositol phosphate metabolism, primary bile acid biosynthesis, phosphatidylinositol signaling system, and linoleic acid metabolism.

Conclusions: The preliminary results suggest that the GC-MS-based method coupled with the pattern recognition method and understanding these cancer-specific alterations 
could make it possible to detect CRC early and aid in the development of additional treatments for the disease, leading to improvements in CRC patients' quality of life.

Keywords: colorectal cancer, metabolomics, GC-MS, cancer tissue and paracarcinoma tissue, preoperative and postoperative serum

\section{INTRODUCTION}

Colorectal cancer (CRC) is a severe health problem and ranks as the third leading cause of tumor-causing death in Europe and the USA (1). And while there are many factors, including the environment, alcohol consumption and smoking are believed to increase the incidence of CRC (1). The pathogenetic progression of colorectal cancer is closely related to polyps (2). Most of the CRCs arise from adenomas, beginning as polyps on the inner wall of the colon or rectum, and subsequently intravasating into lymph vessels or blood vessels, increasing the chance of disseminating to other organs (3). Detecting and removing these malignant polyps promptly is the most effective method at present in CRC prevention. Therefore, the analysis and disposal of malignant polyps is conducive to preventing CRCs.

Currently, there are ways to relatively detect CRCs, such as colonoscopy (4), computed tomography colonography (5), fecal occult blood test (6), and multitarget stool DNA testing (7). However, it has some disadvantages, bleeding risk, inconvenience, no cost-effectiveness, and lower sensitivity and specificity. Unfortunately, most CRC patients are diagnosed when they are in the late stages of the disease with metastasis, making it harder to achieve complete remission. The development of reliable and predictive biomarkers would be a critical tool to identifying individuals with evolving CRC or presence of early disease. However, there is still no tissue or serum biomarker that can be utilized for contented CRC diagnosis. It is urgent to find new screening methods with sensitive, specific, convenient, and non-invasive characters for the early diagnosis of CRC.

Metabolomics is a high-throughput tool useful for exploring metabolites by detecting small-molecule metabolites using mass spectrometry $(<1,800 \mathrm{Da})$. For this reason, small variations in the body can indicate early biological changes to the host due to perturbations in metabolic pathways. Therefore, the metabolome could be regarded as the amplified output of a biological system $(8,9)$. Monitoring fluctuations of certain metabolite levels in body fluids has become an important way to detect early stages in CRC. High-throughput analytical technologies for metabolomics, such as nuclear magnetic resonance (NMR) and mass spectrometry (MS), seem imperative in an untargeted type (10). Recent technological advances allow for the establishment of systematic, holistic methods to relatively short analysis times. In addition, chromatographic methods, such as LC and GC, offer a significant advantage to the MS detection allowing the identification of metabolites based on their chemical properties. Compared to conventional liquid chromatography-tandem mass spectrometry (LC-MS) instruments, gas chromatography-tandem mass spectrometry (GC-MS) detection has gained popularity with its higher chromatographic resolution, reproducibility, and robustness which allowed the establishment of a comprehensive database of identified peaks (11).

Herein, GC-MS-based tissue metabolomics was applied for analyzing the difference between cancer tissue and paracarcinoma tissue in CRC patients. Simultaneously, GCMS-based serum metabolomics between preoperative and postoperative ( 2 weeks) CRC patients is a significant auxiliary for the sake of explicating metabolic pathway transformation and establishing a panel of biomarkers, which would be of diagnostic significance to CRC (Figure 1).

\section{MATERIAL AND METHODS}

\section{Study Population and Sample Collection}

All the experiments of human specimens were in accordance with the ethical code and recommendation issued by the Ethics Committee of Human Experimentation and Chinese Animal Community and with the Helsinki Declaration (approval number: KY2020081). The tissue and serum samples were collected from 48 patients diagnosed with CRC undergoing colorectal resection in Nanjing Hospital of Chinese Medicine Affiliated to Nanjing University of Chinese Medicine. Clinical information, including patient samples, tumor size, and clinical staging, and other information on these CRC patients are shown in Table 1 and Supplemental Information Table S1. The patients we collected came from various localities and owned various living environments and personal habits. Histopathologic examination and immunohistochemistry were utilized to confirm the diagnosis of CRC. Concurrently, the inclusion criteria of the CRC patients in this research included the following: (1) age between 35 and 85 years; (2) conforming to the "Diagnosis, management, and treatment of colorectal cancer" (2015) standard; (3) clear and definite preoperative diagnosis; (4) patients agree to become involved in clinical trials. Exclusion criteria were as follows: (1) gestational period and suckling period of female; (2) malignant hematopathy, including all types of leukemia and anemia; (3) patients with significant metabolic abnormalities; (4) patients with primary tumors in other parts of the body; (5) patients with allergic constitution; (6) use of specific drugs during the last 3 weeks, such as antibiotics, hormones, and nonsteroid anti-inflammatory drugs.

\section{Sample Preparations}

The tissue (cancer tissue and paracarcinoma tissue) and serum (preoperative and postoperative of 2 weeks) samples were screened from 48 CRC volunteers taken from Nanjing Hospital of Chinese Medicine Affiliated to Nanjing University of Chinese Medicine. 


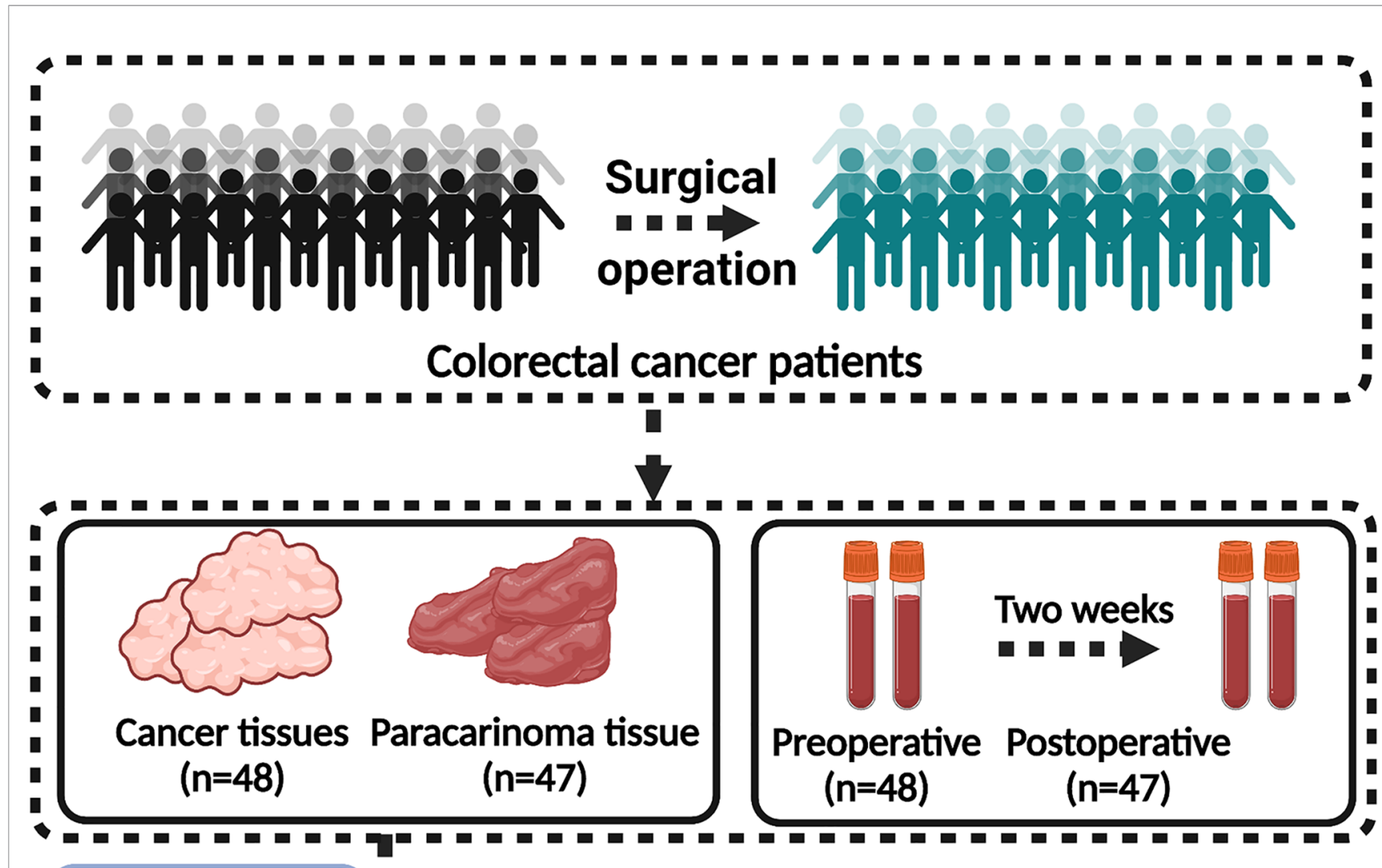

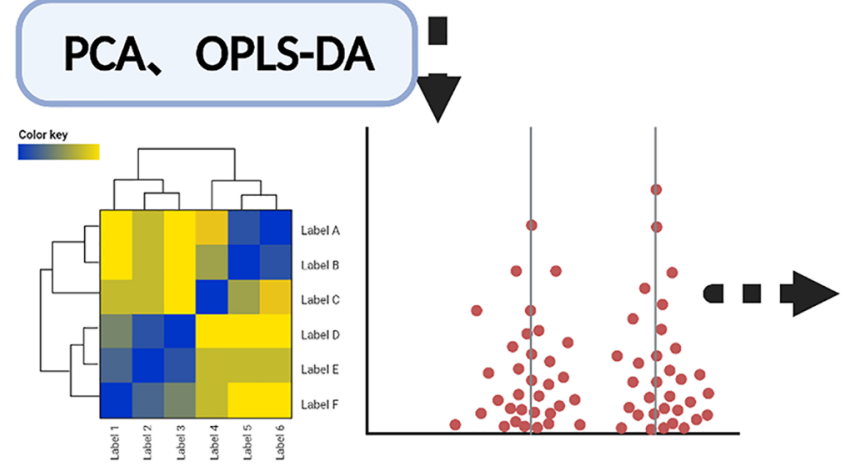

Differential metabilites

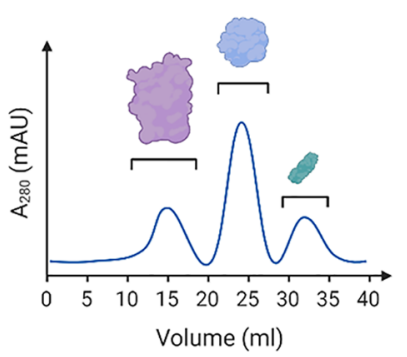

Identification

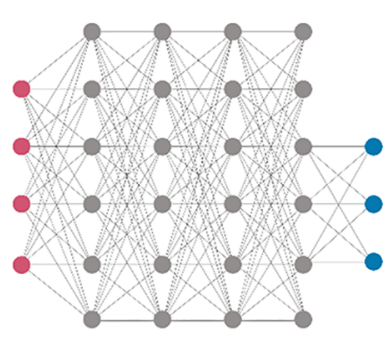

\section{Disturbed metabolic pathways}

FIGURE 1 | The schematic diagram of the experimental design.

After resection of CRC patients, tumor tissue was collected in the central area of solid tumor immediately, and paracancerous tissue was collected in the area about $5 \mathrm{~cm}$ away from the solid tumor. A 100-mg sample was taken and then mixed with $10 \mu \mathrm{l}$ methanol solution that consists of internal standard solution of heptadecanoic acid $(1 \mathrm{mg} / \mathrm{ml})$ into $1.5-\mathrm{ml} \mathrm{EP}$ tubes, extracted with $0.4 \mathrm{ml}$ extraction liquid ( $\mathrm{V}$ (methanol): $\mathrm{V}$ (chloroform) = 3:1), and vortexed for $30 \mathrm{~s}$ at room temperature. Following centrifugation at $13,000 \mathrm{rpm}$ for $10 \mathrm{~min}$ at $4^{\circ} \mathrm{C}$, the resulting $300-\mu \mathrm{l}$ supernatant was transferred into a sample vial for vacuum drying at room temperature. The residue was redissolved in $40 \mu \mathrm{l}$ of a methoxyamine solution (15 $\mathrm{mg} / \mathrm{ml}$ in pyridine) and vortexed for $1 \mathrm{~min}$. An oximation reaction was performed at $37^{\circ} \mathrm{C}$ for $1.5 \mathrm{~h}$. An $80-\mu \mathrm{l}$ aliquot of BSTFA containing $1 \%$ trimethylchlorosilane (TMCS) was then added to the solution, and vortex oscillation for $30 \mathrm{~s}$ followed. The obtained samples were then kept at $70^{\circ} \mathrm{C}$ in an oven for $1 \mathrm{~h}$ before weighing, and a $40-\mu \mathrm{l}$ aliquot of acetonitrile was added. Samples were then centrifuged at 13,200 rpm for $10 \mathrm{~min}$ at $4^{\circ} \mathrm{C}$. The supernatant was transferred to an autosampler vial for GC-MS analysis.

Ten-microliter internal standard solutions (heptadecanoic acid in methanol, $1 \mathrm{mg} / \mathrm{ml}$ ) and extraction liquid (V (methanol): V 
TABLE 1 | Clinical features of colorectal cancer patients enrolled in this study.

\begin{tabular}{|c|c|}
\hline Characteristics & Number \\
\hline Gender (male/female) & $28 / 20$ \\
\hline Age $($ mean $\pm S D)[\min , \max ]$ & $65 \pm 12[36,85]$ \\
\hline Median age among male & 67 \\
\hline Median age among female & 67 \\
\hline Blood glucose (mmol/L) (mean \pm SD) [min, max] & $5.07 \pm 0.63[3.90,6.80]$ \\
\hline Body mass index $\left(\mathrm{kg} / \mathrm{m}^{2}\right)($ mean $\pm \mathrm{SD})[\mathrm{min}, \max ]$ & $22.95 \pm 3.20[17.19,30.39]$ \\
\hline \multicolumn{2}{|l|}{ Tumor sites } \\
\hline Colorectal (number) & 21 \\
\hline Sigmoid colon (number) & 10 \\
\hline Descending colon (number) & 2 \\
\hline Ascending colon (number) & 15 \\
\hline \multicolumn{2}{|l|}{ Pathologic subtypes } \\
\hline Adenocarcinoma (number) & 46 \\
\hline Mucoid carcinoma & 2 \\
\hline Tumor size (cm) [min, max] & $2.5 \times 2 \times 0.8,11 \times 7 \times 3$ \\
\hline \multicolumn{2}{|l|}{ Differentiation } \\
\hline Low & 6 \\
\hline Medium-low & 10 \\
\hline Medium & 31 \\
\hline High & 1 \\
\hline \multicolumn{2}{|l|}{ Infiltration } \\
\hline Adventitia & 11 \\
\hline Deep myometrial & 4 \\
\hline Serosal & 26 \\
\hline Submucosal & 6 \\
\hline Peripheral adipose & 1 \\
\hline \multicolumn{2}{|l|}{ Tumor stage } \\
\hline I & 7 \\
\hline$\|$ & 19 \\
\hline III & 21 \\
\hline IV & 1 \\
\hline
\end{tabular}

$($ chloroform $)=3: 1,600 \mu \mathrm{l})$ were added to serum samples. The mixture was vortexed for $30 \mathrm{~s}$, and the mixture was stored at $37^{\circ} \mathrm{C}$ for $10 \mathrm{~min}$. The resulting $600-\mu \mathrm{l}$ supernatant was transferred into a sample vial for vacuum drying at room temperature. The remaining methods are as aforementioned.

\section{Gas Chromatography-Tandem Mass Spectrometry Conditions}

The samples were analyzed using an Agilent 7890 chromatograph coupled with a 5977B MS system (Agilent Technologies, USA) and EI Source. A DB-5 ms capillary column which was coated with $95 \%$ dimethyl/5\% diphenyl polysiloxane $(30 \mathrm{~m} \times 0.25 \mathrm{~mm}$ inner diameter i.d., $0.25-\mu \mathrm{m}$ film thickness, CA, USA) was utilized in the separation system. The temperature procedure of the column was established as follows: initially, the GC oven temperature was maintained at $60^{\circ} \mathrm{C}$ for $1 \mathrm{~min}$, then the temperature was raised to $325^{\circ} \mathrm{C}$ at a rate of $10^{\circ} \mathrm{C} / \mathrm{min}$ and then maintained at $325^{\circ} \mathrm{C}$ for 10 $\mathrm{min}$. The temperature of the inlet and ion source was maintained at $250^{\circ} \mathrm{C}$, respectively. The injection volume was $1 \mu \mathrm{l}$. Helium was utilized at a constant flow rate of $0.87 \mathrm{ml} / \mathrm{min}$ as the carrier gas. The MS system was operated with electron impact ionization at $70 \mathrm{eV}$ and a scanning range of $\mathrm{m} / \mathrm{z}$ 50-650 (full-scan mode).

\section{Data Processing and Statistical Analysis}

QC samples were analyzed five times at the beginning of the run and injected once after every 10 injections of the random sequenced samples. The raw data obtained from the GC-MS run were transformed to the mzData format using MassHunter Workstation Software (Version B.06.00, Agilent Technologies). Data pretreatments including non-linear retention time alignment, peak discrimination, filtering, alignment, matching, and identification were done using the XCMS Online platform (https://xcmsonline.scripps.edu). The obtained three-dimensional data which were generated from XCMS were conducted by principal component analysis (PCA) and orthogonal partial least square discriminant analysis (OPLS-DA) using SIMCA-P software (version 14.0, Umetrics, Sweden). The t-test was utilized to compare the significant difference between cancer tissue and paracarcinoma tissue for parametric variables. For each statistical analysis, a p-value less than 0.05 was considered as significant. The metabolites with variable importance on the projection (VIP) value greater than 1.0 and $\mathrm{p}$ values less than 0.05 were set for differential metabolites.

\section{Biomarker Identification and Pathway Enrichment Analysis}

Only the variables according with the criteria of "p-value $<0.05$ in ANOVA and VIP values $\geq 1.0$ " were screened as potential biomarkers, and then research for the molecular mechanism was continued. The elemental formula and fragmentation patterns were obtained using MassHunter Workstation Software. Simultaneously, differential metabolites were tentatively identified by library search (NIST for example), Human Metabolome Database (http://www.hmdb.ca/), METLIN (https://metlin.scripps.edu/), and MassBank (http:// www.massbank.jp/). The pathway analysis for metabolites was performed by KEGG using MetaboAnalyst 5.0 (http://www. metaboanalyst.ca/).

\section{RESULTS}

\section{Confirmatory Studies by Histopathologic Examination and Immunohistochemistry}

The CRC samples utilized for immunohistochemistry staining were formalin-fixed, paraffin-embedded tissues and included tissues of the CRC tumor from each patient. Fifty cases of CRC were collected and spotted in duplicate. Diagnostic paraffin blocks were selected on the basis of the availability of suitable formalin-fixed paraffin-embedded tissue. A histological confirmation of CRC was achieved in all cases by a central review using standard tissue sections, and most of the tumor-rich areas were marked in the paraffin blocks. Finally, the CRC was confirmed by hematoxylin and eosin (H\&E) staining (Figure S1).

\section{Result of Multivariate Statistical Analysis}

Typical GC-MS base peak intensity (BPI) chromatograms of tissue samples from the cancer and paracarcinoma and serum samples from the preoperative and postoperative CRC were obtained (Figure 2). Firstly, we specifically compared the tissue 

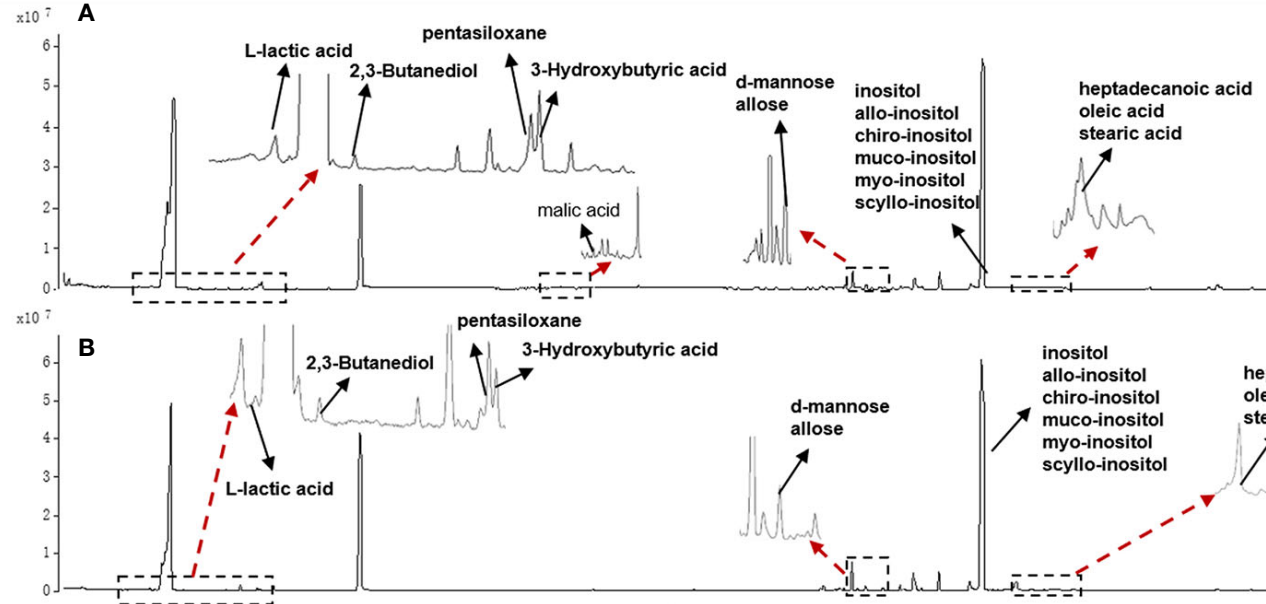

3-Hydroxybutyric acid
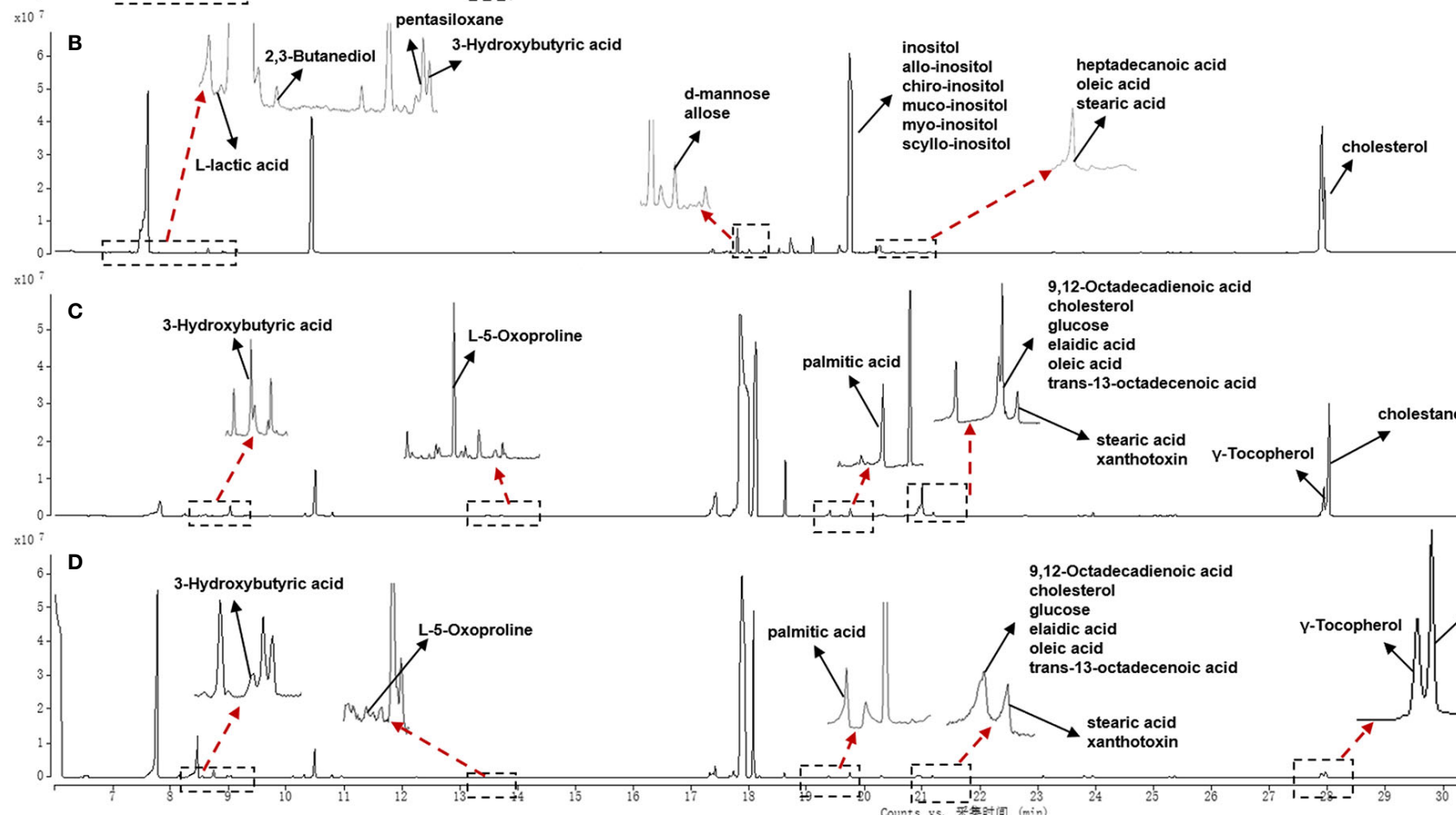

9,12-Octadecadienoic acid

\section{glucose}

glucose
elaic acid

trans-13-octadecenoic acid

stearic acid
cholestane-3,5-diol

xanthotoxin

$y$

FIGURE 2 | Total ion chromatogram of cancer tissue (A) and paracarcinoma tissue (B) and preoperative (C) and postoperative (D) serum of 2 weeks in positive-ion mode. 

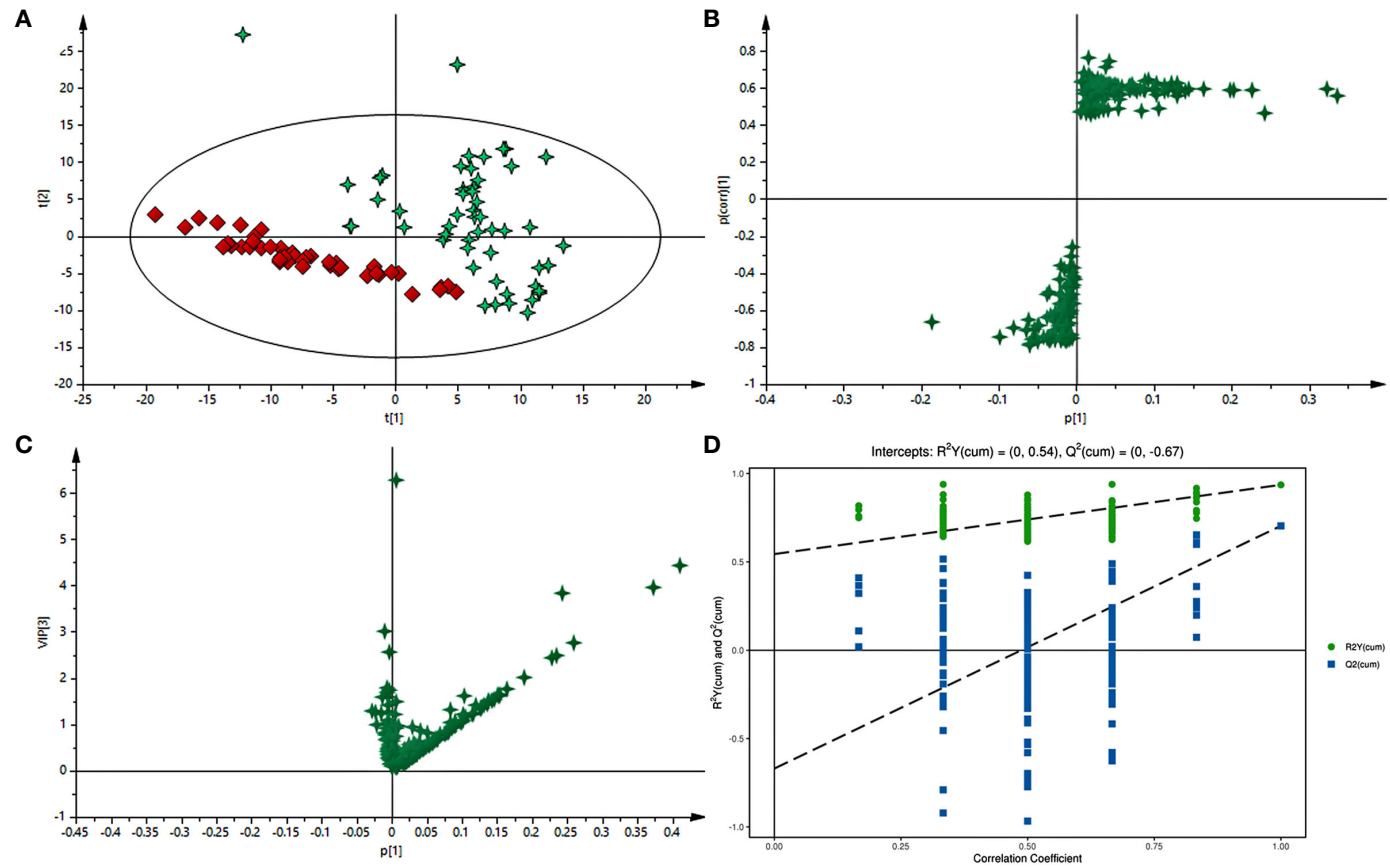

FIGURE 3 | (A) PCA score plot of cancer tissue and paracarcinoma tissue samples in positive-ion mode with the statistical parameters $\left(R^{2} X=0.964, Q^{2}=0.897\right)$. (B) S-plot of OPLS-DA with the statistical parameters in positive-ion mode $\left(R^{2} X=0.940, R^{2} Y=0.756, Q^{2}=0.743\right)$. (C) VIP value plot between cancer tissue and paracarcinoma tissue samples in positive-ion mode. (D) Validation plot of the cancer tissue and paracarcinoma tissue samples in positive-ion mode obtained from 200 permutation tests.
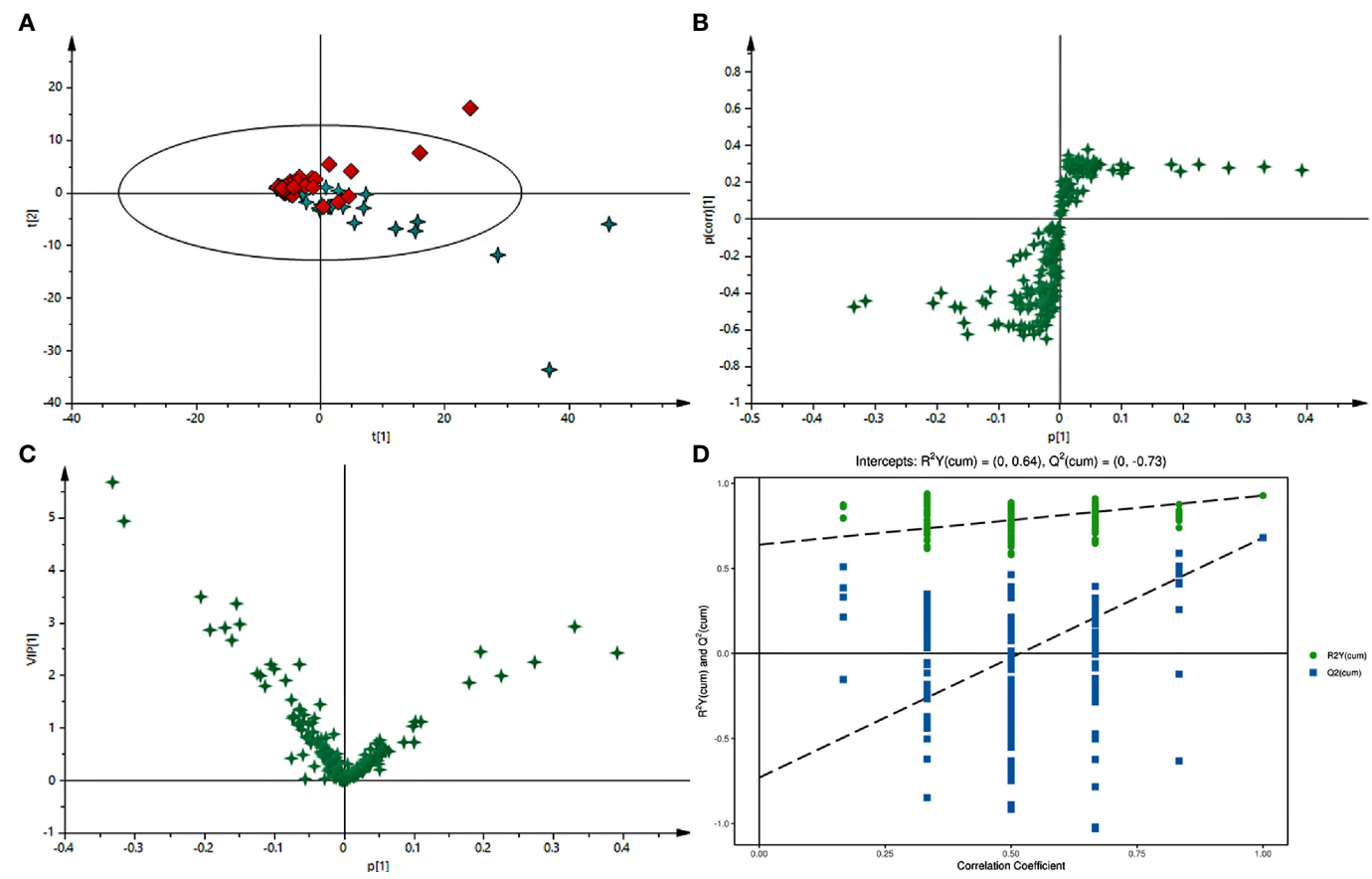

FIGURE 4 | (A) PCA score plot of preoperative and postoperative serum of 2-week samples in positive-ion mode with the statistical parameters $\left(R^{2} X=0.965\right.$, $Q^{2}=$ 0.823). (B) S-plot of OPLS-DA with the statistical parameters in positive-ion mode $\left(R^{2} X=0.939, R^{2} Y=0.639, Q 2=0.844\right)$. (C) VIP value plot between preoperative and postoperative serum of 2-week samples in positive-ion mode. (D) Validation plot of the preoperative and postoperative serum of 2-week samples in positive-ion mode obtained from 200 permutation tests. 
and serum samples for the sake of unambiguous classification. Supervised PCA was conducted on the samples to visualize general clustering, trends, or outliers among the observations. As shown in Figure $\mathbf{3 A}$, the clustering significantly differed between the cancer and paracarcinoma tissues, implying the chemical composition in the significant diversity of tissues between two groups. Simultaneously, the PCA analysis results for serum are shown in Figure 4A. In order to better characterize variations of tissue and serum metabolic profiles, orthogonal partial least-square discriminant analysis was carried out to further identify significantly features/ions. The key differences in $t_{R}-m / z$ pairs were screened by the S-plot and VIP value (Figures 3C and 4C) from OPLS-DA, respectively. In the OPLSDA S-plot (Figures $\mathbf{3 B}$ and $\mathbf{4 B}$ ), each variation point represents an $t_{R}-m / z$ pair; the $X$-axis represents variable contribution which has a further distance from the origin point, the greater the contribution to the separation of groups; the Y-axis represents variable confidence which has a further distance from the origin point, the higher the confidence level of the $t_{R}-m / z$ pairs to the separation of groups (12). The OPLS-DA model was further validated by cross-validation and permutation test (Figures 3D and 4D). Furthermore, the ions with variable importance in the projection (VIP) values $>1.0$ and $p$-value $<0.05$ in ANOVA that at the corner of S-plot were the variables dedicating most to the differences.

\section{Identification of Potential Biomarkers and Pathway Analysis of CRC}

The ions with VIP $\geq 1.0$ and $\mathrm{p}<0.05$ obtained from the S-plot were considered as the candidate putative biomarkers for NIST, METLIN, MetaboAnalyst, and Human Metabolome Database identification. Finally, 17 tissue and 13 serum candidate ions were selected and their corresponding retention time, $\mathrm{p}$-value, $\mathrm{m} / \mathrm{z}$, and VIP value are summarized in Table 2 , and the heat maps of the tissue and serum samples were conducted (Figure 5). Simultaneously, the receiver-operating characteristic (ROC) curve was utilized to evaluate the potential biomarkers (Figure S2). The results show that the trends of stearic acid and cholesterol are consistent, which were the most promising biomarkers.

More detailed analyses of the most relevant pathways and networks for CRC were performed by MetaboAnalyst 5.0, which is a free web-based tool that combines results from powerful pathway enrichment analysis involved in the conditions under study. Metabolic pathway analysis revealed (Table 3 and Figure 6) that 12 pathways contributed to CRC in tissue level, including inositol phosphate metabolism, primary bile acid biosynthesis, steroid biosynthesis, and phosphatidylinositol signaling system. At the same time, there are 12 pathways (Table 3 and Figure 6) contributing to CRC in serum level, including linoleic acid metabolism, primary bile acid

TABLE 2 | The identification of potential biomarkers in tissue level based on GC-MS

\begin{tabular}{|c|c|c|c|c|c|}
\hline Differential metabolites & $\mathrm{m} / \mathbf{z}$ & RT & VIP & Fold change & $\mathbf{p}$ \\
\hline \multicolumn{6}{|c|}{ Cancer tissue and paracarcinoma tissue } \\
\hline L-Lactic acid & 318.0358 & 7.31 & 1.15 & 2.87 & $1.15 \mathrm{E}-10$ \\
\hline 2,3-Butanediol & 116.0217 & 7.78 & 1.16 & 0.19 & 9.97E-06 \\
\hline 3-Hydroxybutyric acid & 373.0875 & 8.94 & 1.26 & 3.07 & $2.43 E-16$ \\
\hline allo-Inositol & 405.1945 & 19.73 & 1.07 & 0.49 & 9.11E-09 \\
\hline Cholesterol & 75.0181 & 27.94 & 1.06 & 0.39 & 1.12E-08 \\
\hline Allose & 97.0683 & 17.98 & 1.21 & 4.02 & $7.61 \mathrm{E}-15$ \\
\hline chiro-Inositol & 381.1673 & 19.73 & 1.05 & 0.51 & 5.93E-08 \\
\hline d-Mannose & 138.0889 & 17.98 & 1.23 & 5.89 & $6.43 E-16$ \\
\hline Heptadecanoic acid & 146.0376 & 20.24 & 1.01 & 0.40 & 3.63E-08 \\
\hline Inositol & 391.1874 & 19.73 & 1.12 & 0.48 & 1.25E-08 \\
\hline Malic acid & 233.0896 & 13.2 & 1.05 & 0.09 & 1.40E-09 \\
\hline Muco-inositol & 407.1886 & 19.73 & 1.01 & 0.53 & 6.86E-08 \\
\hline myo-Inositol & 164.0926 & 19.76 & 1.24 & 9.40 & 7.65E-21 \\
\hline Oleic acid & 117.0078 & 20.24 & 1.02 & 0.39 & 6.86E-08 \\
\hline Pentasiloxane & 354.04859 & 8.92 & 1.26 & 2.94 & 1.05E-11 \\
\hline scyllo-Inositol & 365.1554 & 19.73 & 1.08 & 0.48 & $1.01 \mathrm{E}-08$ \\
\hline Stearic acid & 116.0083 & 20.24 & 1.01 & 0.40 & 1.11E-07 \\
\hline \multicolumn{6}{|c|}{ Preoperative and postoperative serum } \\
\hline Stearic acid & 298.2655 & 21.19 & 1.38 & 1.91 & 1.35E-02 \\
\hline 3-Hydroxybutyric acid & 86.0744 & 8.93 & 2.13 & 5.99 & 2.90E-02 \\
\hline 9,12-Octadecadienoic acid & 264.2632 & 20.98 & 2.24 & 3.63 & $6.28 \mathrm{E}-03$ \\
\hline Cholestane-3,5-diol & 329.3586 & 28.00 & 1.35 & 2.58 & 1.40E-02 \\
\hline Cholesterol & 340.3024 & 20.98 & 2.16 & 3.50 & 4.01E-04 \\
\hline Glucose & 96.0857 & 20.96 & 2.12 & 3.31 & 1.49E-02 \\
\hline elaidic acid & 339.2998 & 20.97 & 2.16 & 3.17 & 8.60E-03 \\
\hline$\gamma$-Tocopherol & 504.4245 & 27.86 & 1.58 & 2.35 & 8.40E-03 \\
\hline L-5-Oxoproline & 258.0370 & 13.73 & 1.92 & 4.68 & 2.11E-03 \\
\hline Oleic acid & 138.1276 & 20.96 & 2.19 & 3.51 & 1.31E-02 \\
\hline Palmitic acid & 285.2381 & 19.40 & 1.44 & 2.04 & 1.60E-02 \\
\hline trans-13-Octadecenoic acid & 84.0748 & 20.97 & 2.11 & 3.10 & 1.69E-02 \\
\hline Xanthotoxin & 356.3373 & 21.18 & 1.06 & 1.78 & 1.33E-02 \\
\hline
\end{tabular}


A

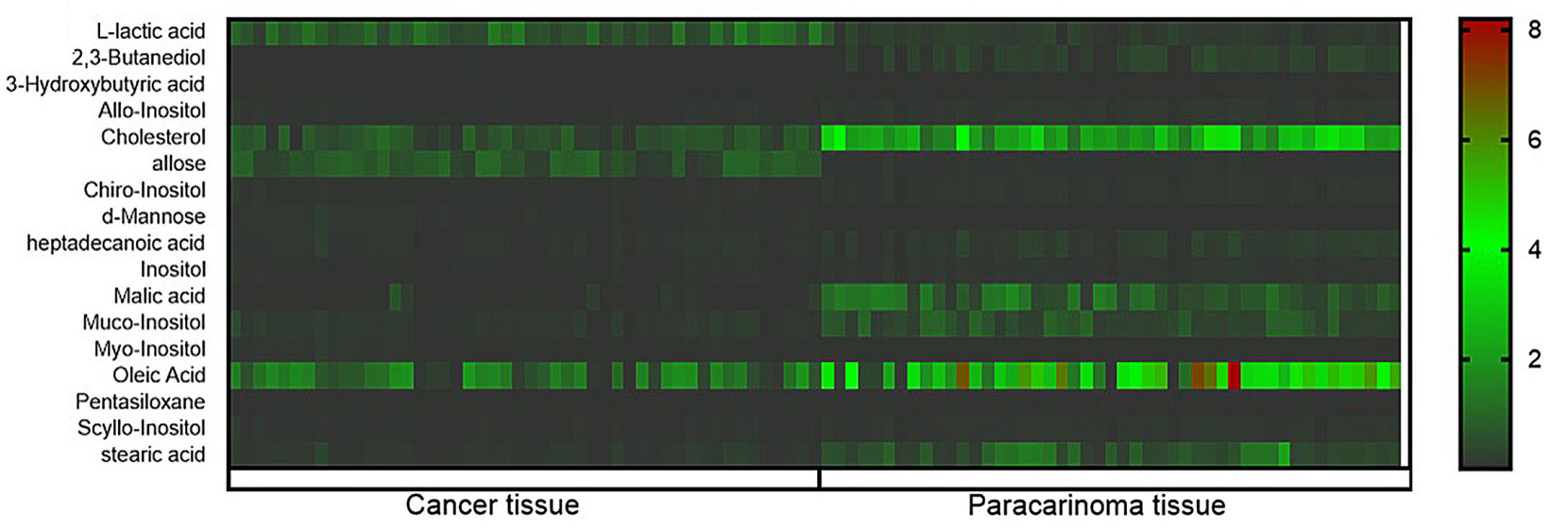

B

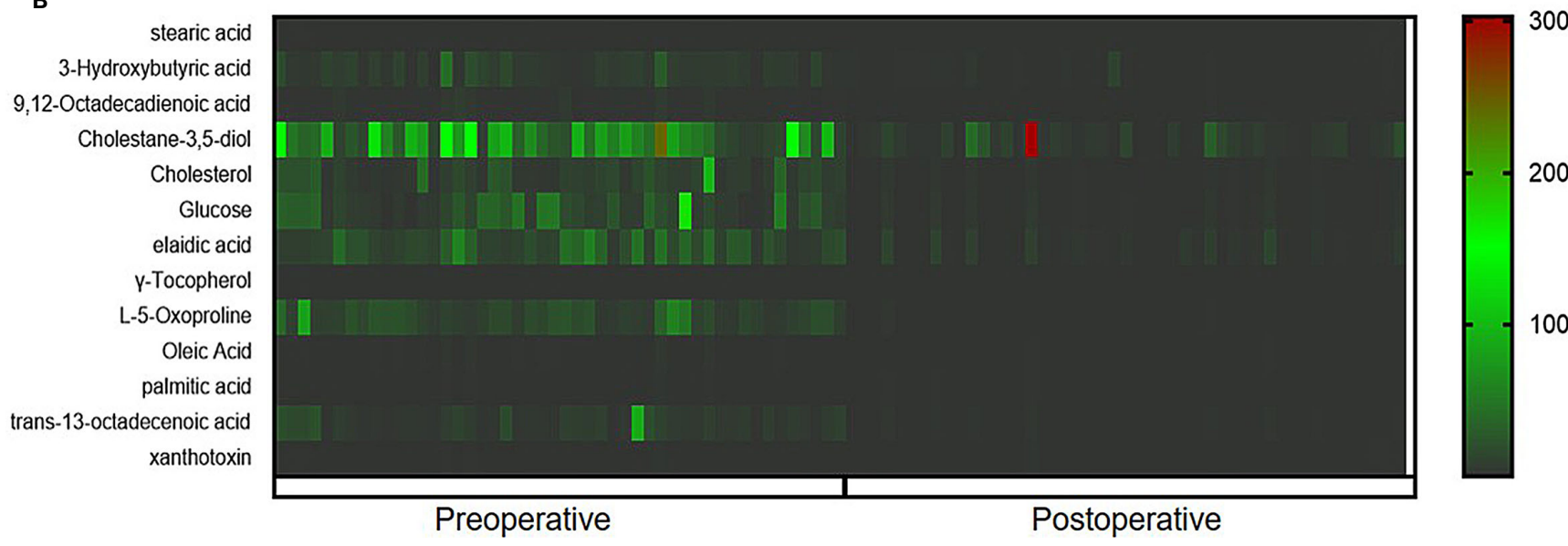

FIGURE 5 | Heatmap of differential metabolites between cancer tissue and paracarcinoma tissue samples (A) and preoperative and postoperative serum of 2-week samples (B).

biosynthesis, and steroid biosynthesis. Ultimately, the most influential pathways contributing to CRC were inositol phosphate metabolism, primary bile acid biosynthesis, phosphatidylinositol signaling system, and linoleic acid metabolism.

\section{Signaling Networks}

Identified potential biomarkers were leading in the KEGG (http://www.kegg.jp/) in finding interactions, which show the relationships among these signal pathways. The networks were primarily related to inositol phosphate metabolism, primary bile acid biosynthesis, steroid biosynthesis, phosphatidylinositol signaling system, and linoleic acid metabolism. According to the pathway flow analysis, the primary bile acid biosynthesis pathway was deemed to be the upstream signaling network. Compared with the paracarcinoma tissue/preoperative serum, the levels of 2,3-butanediol, cholesterol, muco-inositol, oleic acid, and stearic acid were significantly downregulated. Conversely, Llactic acid, allose, cholestane-3,5-diol, and glucose were markedly upregulated (Figure 7).

\section{DISCUSSION}

Colorectal cancer is the third leading cause of cancer-related deaths, and late-stage diagnosis is a major cause of morbidity and mortality of CRC, greatly threatening the health of humans $(13,14)$. The incidence of CRC has been rising continuously in recent decades, resulting in about 900,000 deaths per year globally [15-16]. There is thus an urgent need to discriminate accurate and non-invasive biomarkers to assist the early diagnosis and clinical management of CRC $(15,16)$. However, metabolomics is usually utilized to discriminate the potential biomarkers and, for the purpose of the study, the complexity and hugeness of metabolic networks based on a 
TABLE 3 | Result from ingenuity pathway analysis with MetaboAnalyst 5.0.

\begin{tabular}{|c|c|c|c|c|c|}
\hline Pathway name & Match status & $\mathbf{p}$ & $-\log (p)$ & FDR & Impact \\
\hline \multicolumn{6}{|l|}{ Cancer tissue and paracarcinoma tissue } \\
\hline Biosynthesis of unsaturated fatty acids & $2 / 36$ & 0.025294 & 1.579 & 1 & 0 \\
\hline Synthesis and degradation of ketone bodies & $1 / 5$ & 0.035028 & 1.4556 & 1 & 0 \\
\hline Ascorbate and aldarate metabolism & $1 / 8$ & 0.055506 & 1.2557 & 1 & 0 \\
\hline Butanoate metabolism & $1 / 15$ & 0.10176 & 0.99242 & 1 & 0 \\
\hline Pyruvate metabolism & $1 / 22$ & 0.14595 & 0.83581 & 1 & 0 \\
\hline Glycolysis/gluconeogenesis & $1 / 26$ & 0.1703 & 0.76879 & 1 & 0 \\
\hline Galactose metabolism & $1 / 27$ & 0.17629 & 0.75378 & 1 & 0 \\
\hline Phosphatidylinositol signaling system & $1 / 28$ & 0.18224 & 0.73936 & 1 & 0.03736 \\
\hline Inositol phosphate metabolism & $1 / 30$ & 0.19402 & 0.71216 & 1 & 0.12939 \\
\hline Steroid biosynthesis & $1 / 42$ & 0.26153 & 0.58248 & 1 & 0.0282 \\
\hline Primary bile acid biosynthesis & $1 / 46$ & 0.28286 & 0.54843 & 1 & 0.05065 \\
\hline Steroid hormone biosynthesis & $1 / 85$ & 0.46338 & 0.33406 & 1 & 0.00528 \\
\hline \multicolumn{6}{|l|}{ Preoperative and postoperative serum } \\
\hline Biosynthesis of unsaturated fatty acids & $4 / 36$ & 7.2193E-05 & 4.1415 & 0.0060643 & 0 \\
\hline Synthesis and degradation of ketone bodies & $1 / 5$ & 0.035028 & 1.4556 & 0.98079 & 0 \\
\hline Linoleic acid metabolism & $1 / 5$ & 0.035028 & 1.4556 & 0.98079 & 1 \\
\hline Butanoate metabolism & $1 / 15$ & 0.10176 & 0.99242 & 1 & 0 \\
\hline Glycolysis/gluconeogenesis & $1 / 26$ & 0.1703 & 0.76879 & 1 & 2.10E-04 \\
\hline Glutathione metabolism & $1 / 28$ & 0.18224 & 0.73936 & 1 & 0.00709 \\
\hline Fatty acid elongation & $1 / 39$ & 0.24515 & 0.61057 & 1 & 0 \\
\hline Fatty acid degradation & $1 / 39$ & 0.24515 & 0.61057 & 1 & 0 \\
\hline Steroid biosynthesis & $1 / 42$ & 0.26153 & 0.58248 & 1 & 0.0282 \\
\hline Primary bile acid biosynthesis & $1 / 46$ & 0.28286 & 0.54843 & 1 & 0.05065 \\
\hline Fatty acid biosynthesis & $1 / 47$ & 0.2881 & 0.54045 & 1 & 0.01473 \\
\hline Steroid hormone biosynthesis & $1 / 85$ & 0.46338 & 0.33406 & 1 & 0.00528 \\
\hline Biosynthesis of unsaturated fatty acids & $4 / 36$ & 7.2193E-05 & 4.1415 & 0.0060643 & 0 \\
\hline Synthesis and degradation of ketone bodies & $1 / 5$ & 0.035028 & 1.4556 & 0.98079 & 0 \\
\hline Linoleic acid metabolism & $1 / 5$ & 0.035028 & 1.4556 & 0.98079 & 1 \\
\hline
\end{tabular}

limited number of single pathways to characterize pathological states in animals and human (17).

In this study, the potential mechanisms of CRC, through gas chromatography-tandem mass spectrometry and multivariate statistics analysis, were assessed. A metabolic profiling method based on gas chromatography-tandem mass spectrometry coupled with multivariate statistical analysis including principal component analysis and orthogonal partial least square-discriminant analysis was employed to discriminate the groups, screen differential metabolites, identify the significant metabolites, and illustrate the mechanisms of disease. We gave an illustrative case to show that metabolomics is an innovative method for exploring disease biomarkers or intervention-related perturbed metabolic pathways (18).

In this study, untargeted GC-MS-based tissue (cancer tissue and paracarcinoma tissue) and serum (preoperative and postoperative of 2 weeks) metabolomics was applied to investigate the metabolic state of 50 human subjects. The itemization of CRC consensus molecular subtypes was performed in an effort to explicate the clinical heterogeneity through data analysis results (19). Additionally, an eightbiomarker panel (L-lactic acid, 2,3-butanediol, cholesterol, allose, malic acid, muco-inositol, oleic acid, stearic acid) can differentiate well between cancer tissue and paracarcinoma tissue. A six-biomarker (cholesterol, oleic acid, stearic acid, cholestane-3,5-diol, glucose, 3-hydroxybutyric acid) can differentiate well between the preoperative and postoperative sera of 2 weeks. As a consequence of the above, the levels of 2,3butanediol, cholesterol, muco-inositol, oleic acid, and stearic acid were significantly upregulated. Conversely, L-lactic acid, allose, cholestane-3,5-diol, and glucose were markedly downregulated. CRC is closely related to metabolic dysfunction in the pathway of inositol phosphate metabolism, primary bile acid biosynthesis, steroid biosynthesis, phosphatidylinositol signaling system, and linoleic acid metabolism.

Glucose metabolism was utilized by cancer cells to provide sufficient metabolite precursors and energy to sustain fast cell growth $(20,21)$. Increased glucose uptake and enhanced glycolysis has been identified as a hallmark of cancer cells, with upregulated levels of transporters and enzymes involved in glucose metabolism. In our study, we found that glucose in preoperative CRC serum decreased obviously then that in 2week postoperative serum. The decrease of glucose indicates that glycolysis is a metabolic alteration that appears early on the development of CRC to provide energy for cancer cell growth [22]. Inducing a condition of "hunger" in the cancer cells could be an effective method to improve the disease and possibly even treat the disease (22).

As the molecular skeleton of inositol hexaphosphate (IP6), a carbohydrate, and the precursor of phosphorylated compounds (23), inositol is primarily utilized to treat CRC (24) and other diseases. Inositol exhibits its biological activity on anticancer, 
A

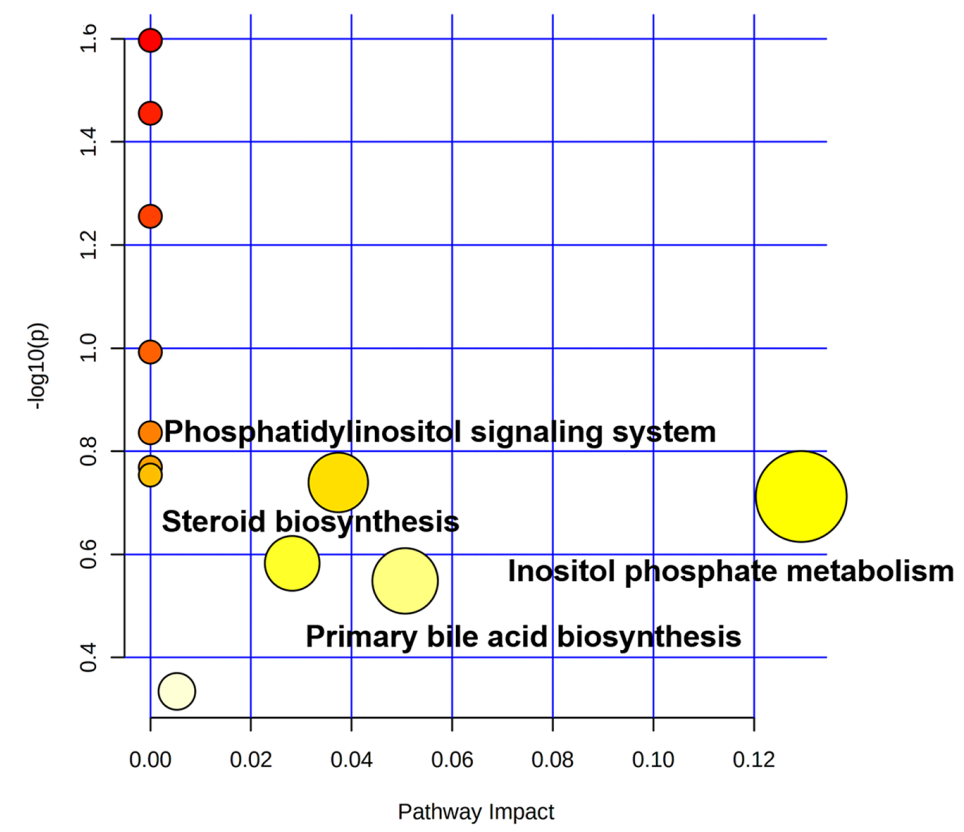

C

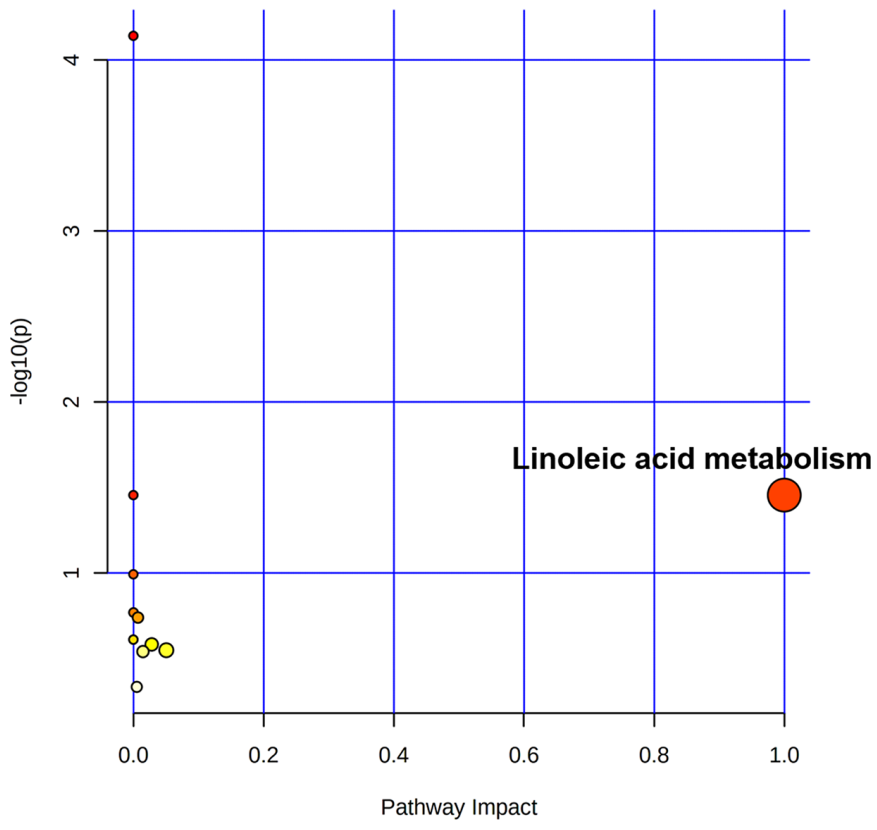

B

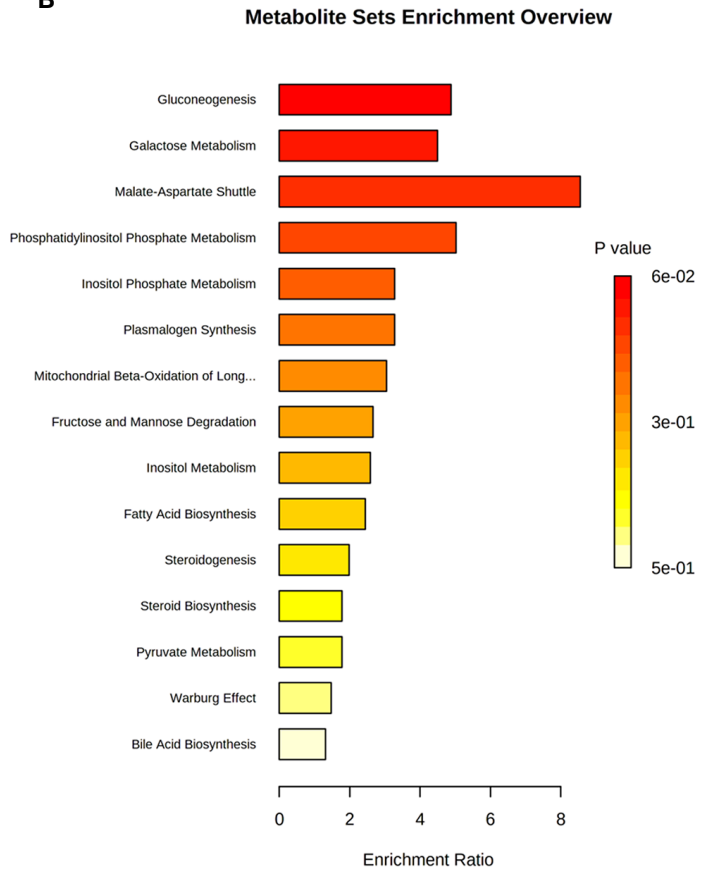

D

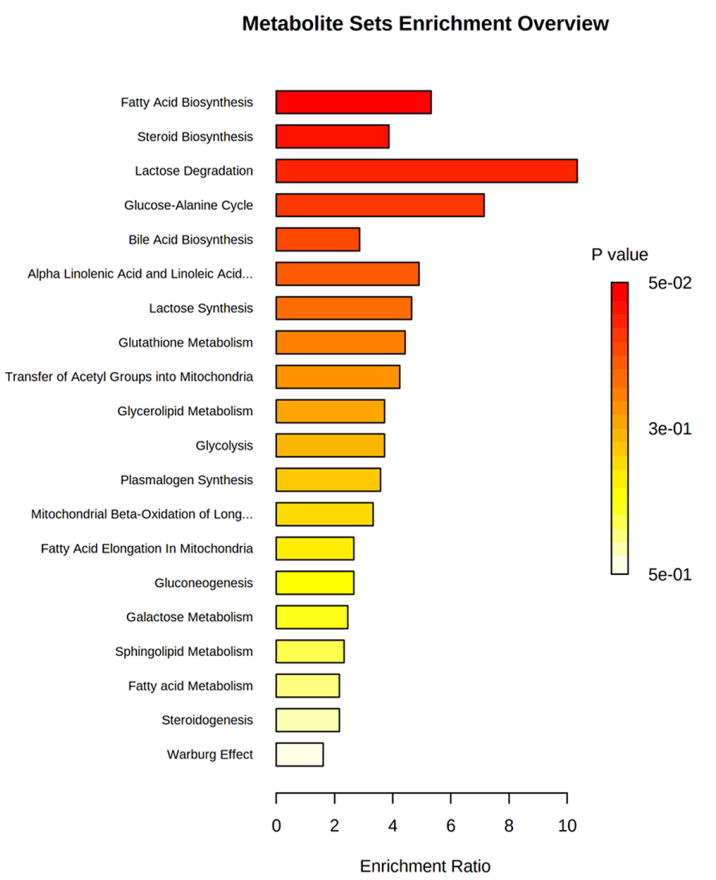

FIGURE 6 | Pathway enrichment analysis of CRC in cancer tissue and paracarcinoma tissue samples (A, B) and preoperative and postoperative serum of 2-week sample (C, D) levels.

and it synergistically reinforces the inhibitory pesticide effects of IP6 on the growth of colon and mammary cancers (24). It was shown that inositol decreased to about $42 \%$ in tumor tissues compared to non-tumor tissues. Simultaneously, with the reduction of inositol, an inhibitor of cancer, the cancer cell grows faster. Therefore, inositol might be a useful tool to inhibit the development and progression of CRC (25).

Although cholesterol is essential in our body, its levels are related with increased CRC risk and the treatment of statin could decrease CRC risk in older adults under 75 years of age 


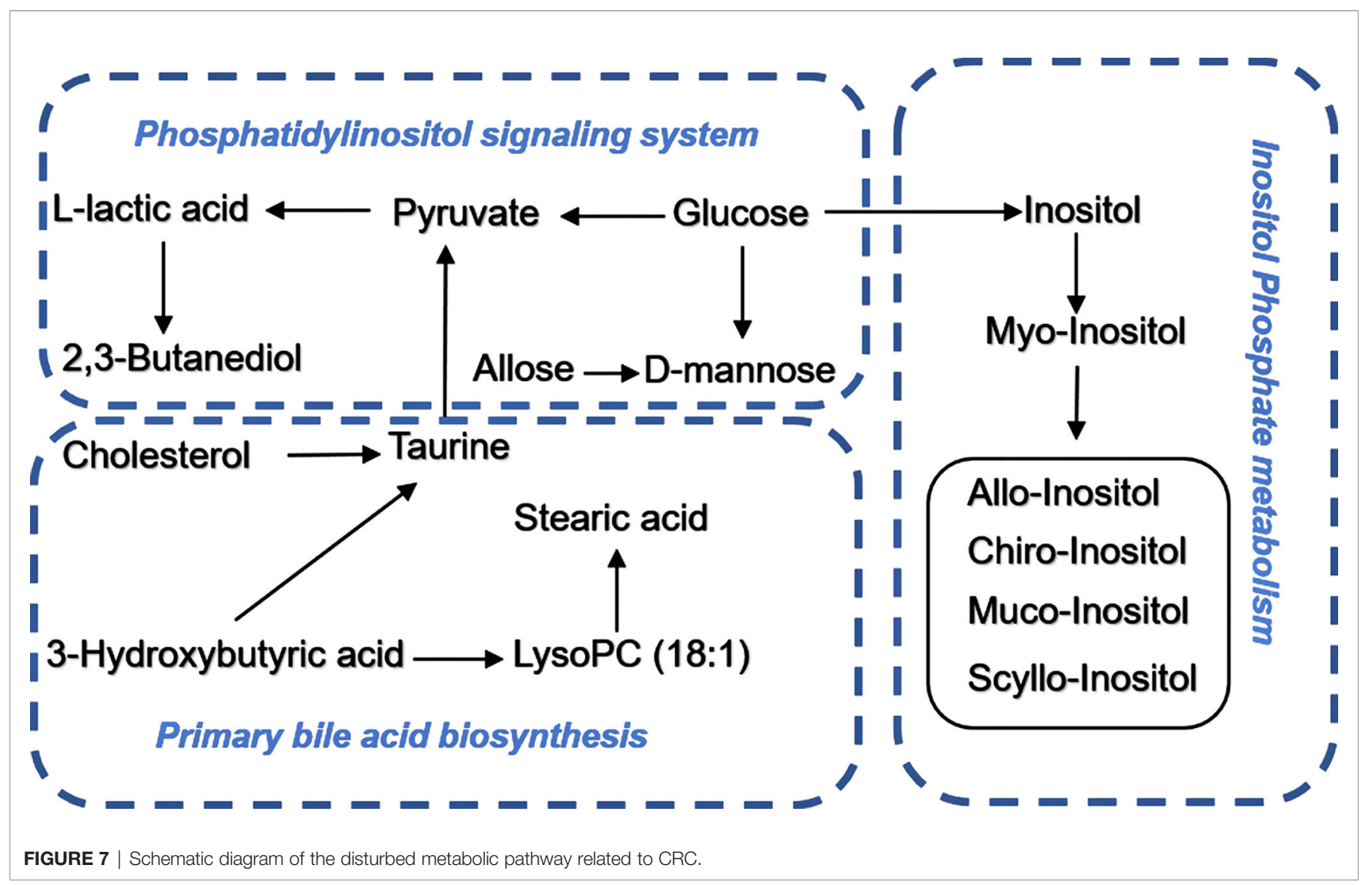

(26). In cholesterol, for example, accumulation is observed in tumors from gastrointestinal cancer patients through increased low-density lipoprotein receptor (LDLR) and decreased ATP-binding cassette transporter (ABCA1) expression (27). Researchers interpreted that the CRC liver metastasis-specific cholesterol metabolic pathway is established for colonization of metastatic CRC cells (27). Simultaneously, inhibiting this CRC liver metastasis-specific cholesterol metabolic pathway could suppress CRC liver metastasis. Finally, it was confirmed that targeting the cholesterol biosynthesis pathway may be a promising therapy for liver metastasis of CRC (28). Cholesterol is an important metabolite participating in bile acid biosynthesis whose levels had increased in the CRC. Simultaneously, bile acids are cholesterol derivatives with detergent properties, are normally seen in the intestine, and have been suggested since 1939 as the tumor-promoting agents (29).

\section{CONCLUSION}

Our research concentrated on the colorectal cancer patients based on untargeted GC-MS-based tissue (cancer tissue and paracarcinoma tissue) and serum (preoperative and postoperative of 2 weeks) metabolomics methods. Finally, 17 tissue and 13 serum candidate ions were selected based on their corresponding retention time, $\mathrm{p}$-value, $\mathrm{m} / \mathrm{z}$, and VIP value. The results show that the trends of stearic acid and cholesterol are consistent, which were the most promising biomarkers. This also means that these metabolites might have important clinical significance for the detection of CRC. Metabolic pathway analysis revealed that 12 pathways contributed to CRC in tissue level, including inositol phosphate metabolism, primary bile acid biosynthesis, steroid biosynthesis, and phosphatidylinositol signaling system. At the same time, there are 12 pathways contributing to CRC in serum level, including linoleic acid metabolism, primary bile acid biosynthesis, and steroid biosynthesis. Ultimately, the most influential pathways contributing to CRC were inositol phosphate metabolism, primary bile acid biosynthesis, phosphatidylinositol signaling system, and linoleic acid metabolism. Further research will be conducted to determine if these biomarkers could be fully integrated into application for early diagnosis of CRC.

\section{DATA AVAILABILITY STATEMENT}

The original contributions presented in the study are included in the article/Supplementary Material. Further inquiries can be directed to the corresponding authors. 


\section{ETHICS STATEMENT}

The studies involving human participants were reviewed and approved by the Nanjing Hospital of Chinese Medicine Affiliated to Nanjing University of Chinese Medicine. The patients/ participants provided their written informed consent to participate in this study.

\section{AUTHOR CONTRIBUTIONS}

GZ, DK, and ZF designed the research. WW, YW, and GZ performed the experiments. BP and FS collected the samples. GZ and WW analyzed the data. GZ, YW, and YZ wrote the manuscript. All authors contributed to the article and approved the submitted version.

\section{REFERENCES}

1. Asante I, Pei H, Zhou E, Liu S, Chui D, Yoo E, et al. Exploratory Metabolomic Study to Identify Blood-Based Biomarkers as a Potential Screen for Colorectal Cancer. Mol Omics (2019) 15(1):21-9. doi: 10.1039/c8mo00158h

2. Jing Y, Wu X, Gao P, Fang Z, Wu J, Wang Q, et al. Rapid Differentiating Colorectal Cancer and Colorectal Polyp Using Dried Blood Spot Mass Spectrometry Metabolomic Approach. IUBMB Life (2017) 69(5):347-54. doi: 10.1002/iub.1617

3. Viswanath B, Kim S, Lee K. Recent Insights Into Nanotechnology Development for Detection and Treatment of Colorectal Cancer. Int J Nanomedicine (2016) 11:2491-504. doi: 10.2147/IJN.S108715

4. Winawer S, Fletcher R, Rex D, Bond J, Burt R, Ferrucci J, et al. Colorectal Cancer Screening and Surveillance: Clinical Guidelines and Rationale-Update Based on New Evidence. Gastroenterology (2003) 124:544-60. doi: 10.1053/gast.2003.50044

5. Simon K. Colorectal Cancer Development and Advances in Screening. Clin. Interv. Aging (2016) 11:967-76. doi: 10.2147/CIA.S109285

6. Center MM, Jemal A, Smith RA, Ward E. Worldwide Variations in Colorectal Cancer. CA Canc J Clin (2009) 59:366-78. doi: 10.3322/caac.20038

7. Imperiale TF, Ransohoff DF, Itzkowitz SH, Levin TR, Lavin P, Lidgard GP, et al. Multitarget Stool DNA Testing for Colorectal-Cancer Screening. N Engl J Med (2014) 371:187-8. doi: 10.1056/NEJMoa1311194

8. Sun H, Zhang AH, Zhang HL, Zhou XH, Wang XQ, Liu L, et al. UltraPerformance Liquid Chromatography/Mass Spectrometry Technology and High-Throughput Metabolomics for Deciphering the Preventive Mechanism of Mirabilite on Colorectal Cancer via the Modulation of Complex Metabolic Networks. RSC Adv (2019) 9:35356. doi: 10.1039/C9RA07687E

9. Zhang A, Sun H, Yan G, Wang P, Han Y, Wang X. Metabolomics in Diagnosis and Biomarker Discovery of Colorectal Cancer. Cancer Lett (2014) 345(1):17-20. doi: 10.1016/j.canlet.2013.11.011

10. Hassan HA, Ammar NM, Serag A, Shaker OG, EI Gendy AN, Abedl-Hamid A. Metabolomics Driven Analysis of Obesity-Linked Colorectal Cancer Patients via GC-MS and Chemometrics: A Pilot Study. Microchemical J (2020) 155:104742. doi: 10.1016/j.microc.2020.104742

11. Zhang J, Tang C, Oberly PJ, Minnigh MB, Achilles SL, Poloyac SM. A Sensitive and Robust UPLC-MS/MS Method for Quantitation of Estrogens and Progestogens in Human Serum. Contraception (2019) 99(4):244-50. doi: 10.1016/j.contraception.2018.12.010

12. Sun H, Wang H, Zhang A, Yan G, Han Y, Li Y, et al. Chemical Discrimination of Cortex Phellodendri Amurensis and Cortex Phellodendri Chinensis by Multivariate Analysis Approach. Pharmacogn Mag (2016) 12(45):41-9. doi: 10.4103/0973-1296.176023

13. Zhang Y, Wu Q, Xu L, Wang H, Liu X, Li S, et al. Sensitive Detection of Colorectal Cancer in Peripheral Blood by a Novel Methylation Assay. Clin Epigenet (2021) 13(1):90. doi: 10.1186/s13148-021-01076-8

14. Wang D, Wang TT, An YB, Jin L, Wang J, Wu GC, et al. Nephroblastoma Overexpressed Protein (NOV) Enhances 5-Fu-Mediated Inhibitory Effect of

\section{FUNDING}

Supported by Nanjing Youth Talent Training Plan of TCM (ZYQ20006), Nanjing Medical Science and Technology Development Project (YKK20167), Training Plan of Clinical Medicine Research Center Jointly Built by Ministry and Province in Nanjing (GCYJZX-2019), and Nanjing Chinese Medicine Modernization and Big Data Research Center, Gu Xiaosong's Academician Workstation.

\section{SUPPLEMENTARY MATERIAL}

The Supplementary Material for this article can be found online at: https://www.frontiersin.org/articles/10.3389/fonc.2021.729512/ full\#supplementary-material

Colorectal Cancer Cell Proliferation via JNK/AP-1/Caspase-8/ Caspase-3 Pathway. Discover Oncol (2021) 12(1):1-9. doi: 10.1007/s12672-021-00403-y

15. Huang X, Lan Y, Li E, Li J, Deng Q, Deng X. Diagnostic Values of MMP-7, MMP-9, MMP-11, TIMP-1, TIMP-2, CEA, and CA19-9 in Patients With Colorectal Cancer. J Int Med Res (2021) 49(5):1-11. doi: 10.1177/ 03000605211012570

16. Arnold M, Sierra MS, Laversanne M, Soerjomataram I, Jemal A, Bray F. Global Patterns and Trends in Colorectal Cancer Incidence and Mortality. Gut (2017) 66:683-91. doi: 10.1136/gutjnl-2015-310912

17. Zhu GX, Feng F. UPLC-MS-Based Metabonomic Analysis of Intervention Effects of Da-Huang-Xiao-Shi Decoction on ANIT-Induced Cholestasis. J Ethnopharmacol (2019) 238:111860. doi: 10.1016/j.jep.2019.111860

18. Leppik L, Kriisa K, Koido K, Koch K, Kajalaid K, Haring L, et al. Profiling of Amino Acids and Their Derivatives Biogenic Amines Before and After Antipsychotic Treatment in First-Episode Psychosis. Front Psychiatry (2018) 24:155(9). doi: 10.3389/fpsyt.2018.00155

19. Koulis C, Yap R, Engel R, Jardé T, Wilkins S, Solon G, et al. Personalized Medicine-Current and Emerging Predictive and Prognostic Biomarkers in Colorectal Cancer. Cancers (Basel) (2020) 12(4):812. doi: 10.3390/ cancers12040812

20. Gu A, Xu J, Ye J, Zhang C. Low NDRG2 Expression Predicts Poor Prognosis in Solid Tumors: A Meta-Analysis of Cohort Study. Med (Baltimore) (2020) 99(41):e22678. doi: 10.1097/MD.0000000000022678

21. Geng HW, Yin FY, Zhang ZF, Gong X, Yang Y. Butyrate Suppresses Glucose Metabolism of Colorectal Cancer Cells via GPR109a-AKT Signaling Pathway and Enhances Chemotherapy. Front Mol Biosci (2021) 8:634874. doi: 10.3389/ fmolb.2021.634874

22. Ning W, Li H, Meng F, Cheng J, Song X, Zhang G, et al. Identification of Differential Metabolic Characteristics Between Tumor and Normal Tissue From Colorectal Cancer Patients by Gas Chromatography-Mass Spectrometry. BioMed Chromatogr (2017) 31(11):e3999. doi: 10.1002/bmc.3999

23. Liu X, Liu C, Chen C, Sun W, Ci Y, Li Q, et al. Combination of Inositol Hexaphosphate and Inositol Inhibits Liver Metastasis of Colorectal Cancer in Mice Through the Wnt/ $\beta$-Catenin Pathway. Onco Targets Ther (2020) 13:3223-35. doi: 10.2147/OTT.S247646

24. Fu M, Song Y, Wen Z, Lu X, Cui L. Inositol Hexaphosphate and Inositol Inhibit Colorectal Cancer Metastasis to the Liver in BALB/c Mice. Nutrients (2016) 8:286-301. doi: 10.3390/nu8050286

25. Ding C, Shan Z, Li M, Chen H, Li X, Jin Z. Characterization of the Fatty Acid Metabolism in Colorectal Cancer to Guide Clinical Therapy. Mol Ther Oncolytics (2021) 20:532-44. doi: 10.1016/j.omto.2021.02.010

26. Han KT, Kim S. Do Cholesterol Levels and Continuity of Statin Use Affect Colorectal Cancer Incidence in Older Adults Under 75 Years of Age? PLoS One (2021) 23: 16(4):e0250716. doi: 10.1371/journal.pone.0250716

27. Jun SY, Brown AJ, Chua NK, Yoon JY, Lee JJ, Yang JO, et al. Reduction of Squalene Epoxidase by Cholesterol Accumulation Accelerates Colorectal 
Cancer Progression and Metastasis. Gastroenterology (2021) 160(4):1194-207. doi: 10.1053/j.gastro.2020.09.009

28. Zhang KL, Zhu WW, Wang SH, Gao C, Pan JJ, Du ZG, et al. Organ-Specific Cholesterol Metabolic Aberration Fuels Liver Metastasis of Colorectal Cancer. Theranostics (2021) 11(13):6560-72. doi: 10.7150/thno.55609

29. Vahabi F, Sadeghi S, Arjmand M, Mirkhani F, Hosseini E, Mehrabanfar M, et al. Staging of Colorectal Cancer Using Serum Metabolomics With 1HNMR Spectroscopy. Iran J Basic Med Sci (2017) 20:835-40. doi: 10.22038/ IJBMS.2017.9022

Conflict of Interest: The authors declare that the research was conducted in the absence of any commercial or financial relationships that could be construed as a potential conflict of interest.
Publisher's Note: All claims expressed in this article are solely those of the authors and do not necessarily represent those of their affiliated organizations, or those of the publisher, the editors and the reviewers. Any product that may be evaluated in this article, or claim that may be made by its manufacturer, is not guaranteed or endorsed by the publisher.

Copyright $\odot 2021$ Zhu, Wang, Wang, Shang, Pei, Zhao, Kong and Fan. This is an open-access article distributed under the terms of the Creative Commons Attribution License (CC BY). The use, distribution or reproduction in other forums is permitted, provided the original author(s) and the copyright owner(s) are credited and that the original publication in this journal is cited, in accordance with accepted academic practice. No use, distribution or reproduction is permitted which does not comply with these terms. 